SLAC-PUB-8208

July 1999

\title{
Surface Impedance and Synchronous Modes *
}

\author{
Gennady Stupakov \\ Stanford Linear Accelerator Center \\ Stanford University, Stanford, CA 94309
}

\begin{abstract}
The concept of the surface impedance is applicable to the case, when obstacles on the surface of a vacuum chamber are small compared to characteristic dimensions of the problem. We apply this concept to the calculation of a synchronous mode that can propagate in a tube with slightly corrugated walls. We also show that such a mode can propagate in a pipe with a rough surface, or a perforated pipe with a large number of holes.
\end{abstract}

Presented at the Workshop on Instabilities of High Intensity Hadron Beams in Rings, Brookhaven National Laboratory, June 28 to July 1, 1999

${ }^{*}$ Work supported by Department of Energy contract DE-AC03-76SF00515 


\section{Introduction}

There are cases in practice, when the impedance of a vacuum chamber of accelerator is determined by a small obstacle located on the surface of the wall. We call an obstacle small if its characteristic size $g$ is much smaller then both the transverse size of the chamber $a$ and the wavelength $\lambda / 2 \pi$ associated with the frequency $\omega$ for which the impedance is calculated. For the case of a single small axisymmetric obstacle, a general treatment of the problem was given in Ref. [1]. It was shown that in this case the obstacle is characterized by an electric and magnetic moments, which can be found from the solution of the respective electrostatic and magnetostatic problems, and both contribute to the interaction with the beam.

There are situations however, when many small obstacles densely populate the surface of the wall. Examples are: a rough perfectly conducting surface with a random profile of roughness [2, 3]; a corrugated wall of vacuum bellows; and a wall perforated by a large number of small pumping holes [4]. To describe the interaction of such wall with the electromagnetic field, on can introduce an averaged characteristic of the surface in terms of the surface impedance $\zeta(\omega)$. If this surface impedance is known, it is easy to calculate the longitudinal and transverse impedances for the beam propagating in the chamber.

The concept of the surface impedance has been previously used by $\mathrm{V}$. Balbekov $[5,6]$ in the treatment of small obstacles on the pipe surface. More recently, M. Dohlus discussed the application of this concept to the calculation of the impedance of rough surfaces [7].

In this paper we will consider how the concept of surface impedance can be used to simplify calculations of the impedance for an ultrarelativistic beam. Following [7], we will show how the surface impedance can explain the appearance of a synchronous wave that propagates with the phase velocity equal to the speed of light, and can be excited by a relativistic beam. We will also apply this concept to a rough surface, and, directly from Maxwell's equations, will find $\zeta$ for a corrugated wall in the limit of shallow corrugations. For simplicity, in this paper, we will consider the longitudinal impedance only, although most of our results can be easily generalized for the transverse impedance as well. 


\section{Longitudinal impedance of ultrarelativistic beam in circular tube}

Consider an infinitely thin beam, traveling with the speed of light along the axis of a circular pipe of radius $a$. The only nonzero components of the fields generated by the beam are $E_{r}(r, z, t), E_{z}(r, z, t)$, and $H_{\theta}(r, z, t)$, where $r$ and $z$ are cylindrical coordinates. We want to calculate the longitudinal impedance for such beam at frequency $\omega$.

In Fourier representation, the beam current is given by $I(z, t)=I_{0} e^{-i \omega t+i k z}$, where $k=\omega / c$, corresponding to $v=c$. The Maxwell's equations in vacuum, that is for $0<r<a$, are

$$
\nabla \times \boldsymbol{H}=-\frac{i \omega}{c} \boldsymbol{E}, \quad \nabla \times \boldsymbol{E}=\frac{i \omega}{c} \boldsymbol{H} .
$$

It follows from Eqs. (1) that

$$
\frac{\partial}{\partial r} \frac{1}{r} \frac{\partial}{\partial r} r H_{\theta}=0
$$

whose solution is

$$
H_{\theta}=\frac{A}{r}+\frac{1}{2} B r
$$

where $A$ and $B$ are unknown constants. Using the relation

$$
E_{z}=\frac{i}{k}(\nabla \times \boldsymbol{H})_{z}=\frac{i}{k} \frac{1}{r} \frac{\partial}{\partial r} r H_{\theta}
$$

one finds from Eq. (3) $E_{z}=i B / k$. It is important to emphasize here the the longitudinal electric field in the pipe does not depends on radius $r$ (this is only true in the limit $v=c$ ).

Using Ampere's law, one can relate the constant $A$ to the beam current, $A=2 I_{0} / c$, and express the magnetic field in the pipe in terms of the (yet unknown) longitudinal electric field

$$
H_{\theta}=\frac{2 I_{0}}{r c}+E_{z} \frac{k r}{2 i}
$$

We see that the whole solution is now determined by a single constant the electric field $E_{z}$. To find this constant, we need to specify a boundary condition on the surface of the pipe. 
After the problem is solved, we can find the longitudinal impedance per unit length of the pipe with the help of the following relation

$$
Z=-\frac{E_{z}}{I_{0}} .
$$

\section{Surface impedance}

There are situations when the longitudinal electric field on the surface of the pipe can be directly related to the magnetic field $H_{\theta}$ at the same point. A well known example of such relation is the Leontovich boundary condition [8] on the surface of the metal when the skin depth associated with the frequency $\omega$ is much smaller than the tube thickness.

In general case, let us assume that a relation between the longitudinal electric field and the tangential magnetic field holds on the wall, $r=a$,

$$
\left.E_{z}\right|_{r=a}=-\left.\zeta(\omega) H_{\theta}\right|_{r=a},
$$

where the complex variable $\zeta(\omega)$ is the surface impedance of the tube ${ }^{\dagger}$. For the case of the Leontovich boundary condition (see [8], Eq. $(87,6)) \zeta=$ $(1-i) \sqrt{\omega / 8 \pi \sigma}$, where $\sigma$ is the conductivity of the metal.

Using Eq. (7) we can now solve equation (5) to obtain

$$
E_{z}=-\zeta \frac{2 I_{0}}{a c} \frac{1}{1+k a \zeta / 2 i} .
$$

Let us consider first a situation when the second term in the denominator of Eq. (8) can be neglected, which is valid for small frequencies, $k a \ll|\zeta|^{-1}$. In this case, neglecting the second term in the denominator of Eq. (8) yields

$$
E_{z}=-\zeta \frac{2 I_{0}}{a c}
$$

and the longitudinal impedance becomes

$$
Z=-\frac{E_{z}}{I_{0}}=\frac{2 \zeta}{a c}=\frac{Z_{0} \zeta}{2 \pi a} .
$$

${ }^{\dagger}$ In the general case, $\zeta$ is a function of two variables, $\zeta=\zeta(k, \omega)$. In this paper, however, we consider only the application of $\zeta$ to ultrarelativistic beams, when $k=\omega / c$, and hence $\zeta$ can be considered as a function of frequency. 
For the conducting wall, using the Leontovich boundary condition, one immediately obtains the well known result $Z=(1-i) \sqrt{\omega / 2 \pi \sigma} / a c$.

As a second example, consider a thin dialectric/magnetic layer of thickness $h \ll a$ covering the surface of a perfectly conducting metal [9, 10]. As it follows from the Maxwell's equation, inside the layer the following equation holds

$$
\frac{\partial E_{z}}{\partial r}=i k\left(\epsilon^{-1}-\mu\right) H_{\theta}
$$

where $\epsilon$ is the dielectric constant, and $\mu$ is the magnetic permeability of the layer. Because the layer is thin, we can integrate this equation using the boundary condition on the surface of the metal, $\left.E_{z}\right|_{r=a}=0$, and assuming that the magnetic field is approximately constant within the layer,

$$
\left.E_{z}\right|_{r=a-h}=-i k h\left(\epsilon^{-1}-\mu\right) H_{\theta} .
$$

Since in vacuum $E_{z}$ does not depend on $r$, we can move the boundary condition (12) from $a-h$ to $a$ and impose it on the surface of the wall - this will produce a small error of the order of $h / a$. Eq. (12) is then equivalent to the surface impedance that is purely imaginary with the negative imaginary part (assuming $\epsilon, \mu>1$ ),

$$
\zeta=-i k h\left(\mu-\epsilon^{-1}\right) .
$$

In the small-frequency approximation this gives the inductive impedance (per unit length of the pipe), $Z=-i L \omega$, where

$$
L=\frac{2 h}{b c^{2}}\left(\mu-\epsilon^{-1}\right) .
$$

\section{Synchronous mode}

If we do not neglect the second term in the denominator of Eq. (5), we find for the impedance the following formula

$$
Z(\omega)=\frac{2 \zeta(\omega)}{a c} \frac{1}{1+k a \zeta / 2 i} .
$$

An important feature of this impedance is that for a purely imaginary $\zeta$ with

$\operatorname{Im} \zeta<0$, there is a singularity at the frequency $\omega=\omega_{0}$ that satisfies the following equation

$$
\omega_{0} \zeta\left(\omega_{0}\right)=-\frac{2 i c}{a}
$$


This singularity is due to the wave that can propagate in the tube with the phase velocity equal to the speed of light. Indeed, it is easy to check by going back to the derivation of Sec. 1, that if Eq. (16) is satisfied, an electromagnetic wave

$$
E_{r}=H_{\theta}=H_{0} \frac{r}{a} e^{-i \omega_{0} t+i k z}, \quad E_{z}=H_{0} \frac{2 i}{k a} e^{-i \omega_{0} t+i k z},
$$

satisfies both the Maxwell's equations and the boundary condition (7). For a thin dielectric layer, with $\zeta$ given by Eq. (13), $\omega_{0}=c \sqrt{2 / a h\left(\mu-\epsilon^{-1}\right)}$.

This mode will be excited by the beam resulting in the wakefield that can be found by integration of the contribution from the pole of the impedance

$$
w(s)=\frac{2 Z_{0} c}{\pi a^{2}} \frac{\zeta\left(\omega_{0}\right)}{\left.(\omega \zeta)^{\prime}\right|_{\omega=\omega_{0}}} \cos \left(\omega_{0} s / c\right) .
$$

In the case of linear dependence, $\zeta \propto \omega$, Eq. (18) reduces to $w(s)=$ $\left(Z_{0} c / \pi a^{2}\right) \cos \left(\omega_{0} s / c\right)$.

\section{Rough surface}

In a simple model [2], a rough surface can be considered as a collection of small densely packed bumps sitting on a plane perfectly conducting substance. As was pointed out in [1], a small obstacle on the surface is characterized by dipole and magnetic moments. A dense collection of such obstacles can be considered as an artificial dielectric layer, if the inverse wavenumber $k^{-1}$ is much larger then the size of obstacles (bumps). Not surprisingly, it was found that, similar to the dielectric layer, the rough surface is characterized by an inductive impedance, and can support propagation of a synchronous wave $[11,12]$.

A quantitative theory of the rough wall impedance, applicable (within some limitations) for arbitrary random profile, was developed in Ref. [13]. The approach is based on the assumption that the angle between the normal to the rough surface and the radial direction is small compared to unity. If we assume that the rough surface is given by the equation $y=h(x, z)$, then the small-angle approximation means that $|\nabla h| \ll 1$. It is also required that the height of the bumps and their characteristic width $g$ be small compared to the radius of the tube $b, g,|h| \ll b$, and the frequency $\omega$ is small compared to $c / g, \omega \ll c / g$. 
To describe a rough surface with a random profile, we assume that $h(x, y)$ is a random function with zero average, $\langle h(x, z)\rangle=0$. Statistical properties of such a surface are characterized by the correlation function $K(x, y)$,

$$
K\left(x-x^{\prime}, z-z^{\prime}\right)=\left\langle h\left(x^{\prime}, z^{\prime}\right) h(x, z)\right\rangle,
$$

where the angular brackets denote averaging over possible realizations of $h(x, z)$. Equation (19) implies that statistical properties of $h(x, z)$ do not depend on the position on the surface. An important statistical characteristic of the roughness is the spectral density (or spectrum) $R\left(\kappa_{z}, \kappa_{x}\right)$, defined as a Fourier transform of the correlation function,

$$
R\left(\kappa_{x}, \kappa_{z}\right)=\frac{1}{(2 \pi)^{2}} \int d x d z K(x, z) e^{-i \kappa_{x} x-i \kappa_{z} z} .
$$

The main result of Ref. [13] is that the longitudinal impedance (per unit length) of a circular tube of radius $a$ with a rough perfectly conducting surface characterized by the spectral function $R\left(\kappa_{x}, \kappa_{z}\right)$ in the frequency range limited by the condition $\omega \ll c / g$, is $Z(\omega)=-i \omega L_{\mathrm{r} s}$ with the inductance

$$
L_{\mathrm{r} s}=\frac{Z_{0}}{2 \pi c a} \int d \kappa_{z} d \kappa_{x} R\left(\kappa_{x}, \kappa_{z}\right) \frac{\kappa_{z}^{2}}{\kappa} .
$$

The presence of the factor $\kappa_{z}^{2}$ in the integrand of Eq. (21) means that the contributions to $L_{\mathrm{rs}}$ of roughness in longitudinal $(z)$ and azimuthal $(x)$ directions are different. For example, bellow-type variations on the surface have spectral components with $\kappa_{z} \neq 0$ and $\kappa_{x}=0$, and result in nonvanishing $L_{\mathrm{r} s}$. On the other hand, grooves of all sizes in the longitudinal direction, as described in the previous Section, generate a spectrum with $\kappa_{x} \neq 0$ and $\kappa_{z}=0$, and according to Eq. (21) do not contribute to $L_{\mathrm{r} s}$.

Using Eq. (10), valid in the limit of small frequencies, we can infer the roughness surface impedance $\zeta_{\text {rs }}$ from Eq. (21)

$$
\zeta_{\mathrm{r} s}=-i k \int d \kappa_{z} d \kappa_{x} R\left(\kappa_{x}, \kappa_{z}\right) \frac{\kappa_{z}^{2}}{\kappa} .
$$

As was pointed out in the previous section, this kind of impedance allows for the propagation of a synchronous mode at some frequency $\omega_{0}$. Indeed, such a mode was found in Ref. [11] (see also [12]), where its existence was explained in analogy with a mode in a pipe coated by a dielectric layer. 


\section{Surface Impedance for Corrugated Pipe}

In a simple case of a corrugated pipe, the surface impedance can be found directly from Maxwell's equations, even when the wavelength is compared to the period of corrugation.

Let us assume that the pipe surface is given by

$$
r=a-h \sin \kappa z
$$

where $2 \pi / \kappa$ is the period of corrugation, and $h$ is its amplitude. We assume that both the wavelength and the amplitude are small, $h \ll a$ and $\kappa a \ll 1$. This allows us to neglect the curvature effects and to consider the surface locally as a plane one. We will also assume a shallow corrugation

$$
h \kappa \ll 1 \text {, }
$$

that is the amplitude of the bumps is much smaller then their period.

Introducing a local Cartesian coordinate system $x, y, z$ with $y=a-r$ (directed from the wall toward the beam axis), and $x$ directed along $\theta$, the surface equation becomes $y=y_{0}(z) \equiv h \sin \kappa z$. The magnetic field near the surface $H_{x}(y, z)$ does not depend on $x$ (that is $\theta$ ) due to the axisymmetry of the problem. It satisfies the Helmholtz equation

$$
\frac{\partial^{2} H_{x}}{\partial y^{2}}+\frac{\partial^{2} H_{x}}{\partial z^{2}}+k^{2} H_{x}=0
$$

with the boundary condition

$$
\left.(\boldsymbol{n} \nabla H)\right|_{y=y_{0}}=0,
$$

where $\boldsymbol{n}$ is the normal vector to the surface, $\boldsymbol{n}=(0,1,-h \kappa \cos \kappa z)$.

Note that the longitudinal electric field $E_{z}$ can be expressed in terms of $H_{x}$,

$$
E_{z}=-\frac{i}{k} \frac{\partial H_{x}}{\partial y}
$$

Using the small parameter $h / a$, we will develop a perturbation theory for calculation of $H_{x}$ near the surface and find how $E_{z}$ is related to $H_{x}$.

In the zeroth approximation, the $z$ dependence of $H_{x}$ is dictated by the beam current periodicity,

$$
H_{x}(y, z)=\mathcal{H}(y) e^{i k z} .
$$


Putting Eq. (28) into Eq. (25) we find that $d^{2} \mathcal{H} / d y^{2}=0$, hence $\mathcal{H}(y)=$ $H_{0}+A y$, where the constant $A$ can be related, through Eq. (27), to the electric field on the surface, and hence $\zeta, A=i k E_{z}=-i k \zeta H_{0}$. We will see below that $A$ is of the second order in $h$.

For a flat surface, for which $\boldsymbol{n}=(0,1,0)$, from the boundary condition (26), we would conclude that $A=0$, however, the corrugations result in a nonzero $A$, and hence $E_{z}$. Substituting the magnetic field (28) into the right hand side of Eq. (26) one finds

$$
\boldsymbol{n} \nabla H=-\frac{1}{2} i h k \kappa H_{0}\left[e^{i(k+\kappa) z}-e^{i(k-\kappa) z}\right]-i k \zeta H_{0} e^{i k x} .
$$

Clearly, the boundary condition is not satisfied in this approximation. To correct this, we have to add satellite modes to the fundamental solution (28)

$$
H_{x}(y, z)=\mathcal{H}(y) e^{i k z}+\mathcal{H}_{1}(y, z)
$$

where

$$
\mathcal{H}_{1}(y, z)=B^{+}(y) e^{i(k+\kappa) z}+B^{-}(y) e^{i(k-\kappa) z} .
$$

The dependence of $B^{ \pm}$versus $y$ can be found from the Helmholtz equation,

$$
B=B_{0}^{ \pm} e^{-y \sqrt{\kappa^{2} \pm 2 \kappa k}},
$$

where $B_{0}^{ \pm}$are constants. In order for $B^{ \pm}$to exponentially decay in $y$, we have to assume here that $k<\kappa / 2$.

Substituting $\mathcal{H}_{1}$ terms into the boundary condition (26) generates first order terms that have $x$-dependence $\exp i(k \pm \kappa) x$, and second order terms proportional to $\exp (i k x)$. From the former one finds that

$$
B_{0}^{ \pm}=-\frac{i k \kappa H_{0} h}{2 \sqrt{\kappa^{2} \pm 2 k \kappa}}
$$

and the latter gives an expression for the surface impedance $\zeta$,

$$
\zeta(k)=-\frac{1}{4} i k h^{2} \kappa \frac{\sqrt{\kappa^{2}+2 k \kappa}+\sqrt{\kappa^{2}-2 k \kappa}}{\sqrt{\kappa^{2}-4 k^{2}}} .
$$




\section{Synchronous wave in corrugated pipe}

First, consider the case of small frequencies, $k \ll \kappa$. In this limit, Eq. (34) reduces to

$$
\zeta=-\frac{1}{2} i k h^{2} \kappa
$$

Being purely imaginary with the negative imaginary part, this $\zeta$ allows for a propagation of the synchronous mode. Its frequency is given by Eq. (16)

$$
\omega_{0}=\frac{2 c}{h \sqrt{a \kappa}} .
$$

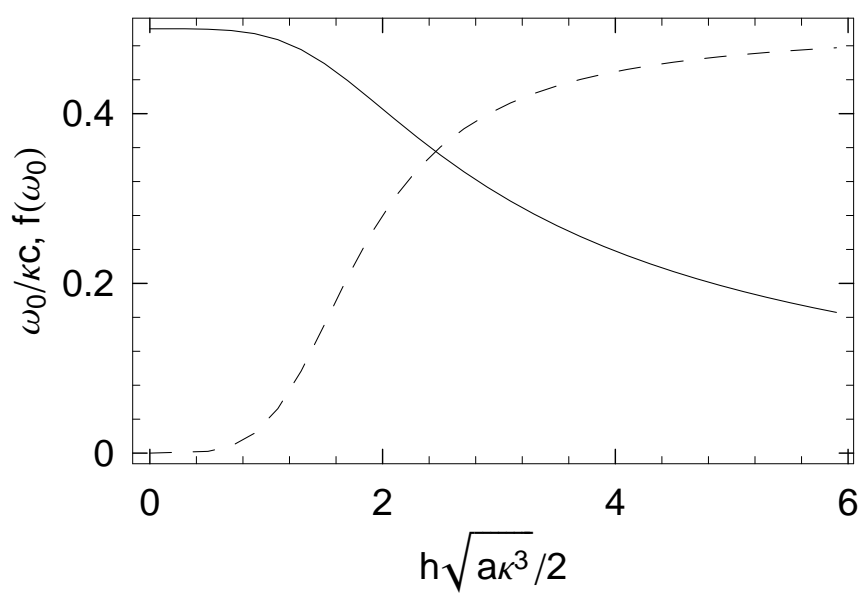

Figure 1: Frequency $\omega_{0}$ (solid curve) and the loss form-factor $f$ (dashed curve) for the synchronous mode as a function of height $h$.

For the general case, when $k$ can be comparable with $\kappa$, one has to solve the exact dispersion relation. The result is shown in Fig. 1, where we also plot the formfactor $f$ that gives the wakefield of the mode $w(s)=$ $\left(2 f Z_{0} c / \pi a^{2}\right) \cos \left(\omega_{0} s / c\right)$. We see that decreasing the height of the corrugation results in smaller wakes, and hence leads to the suppression of the interaction of the synchronous wave with the beam.

We have to mention here that our theory breaks down for very small values of $h$. Indeed, we implicitly assumed that the satellite harmonics in Eq. (32) are localized near the surface, otherwise our approximation of plain 
surface becomes invalid. Hence, we have to require that $\kappa-2 k \gg a^{-1}$, which gives the following condition of applicability

$$
h>a^{-1 / 4} \kappa^{-5 / 4} .
$$

This condition explains why this mode has not been found in earlier papers $[14,15]$ that treated the problem of a corrugated pipe: being purturbative in parameter $h$ the approach developed in those papers is applicable only when $h$ can be made arbitrarily small.

\section{Perforated pipe}

The notion of the surface impedance can be also applied to the case of a pipe with a large number of small holes perforated in the pipe wall. Again, we have to assume that the wavelength $c / \omega$ is much larger then the hole size $b$ and the average distance between the holes $d, c / \omega \gg b, d$.

The value of $\zeta$ can be found from the correspondence between magnetic and electric properties of a thin dielectric layer considered in Section III and a large collection of small holes.

Indeed, the electric polarizability $\alpha_{e}$ of a single round hole of radius $b$ is $\alpha_{e}=-b^{3} / 3 \pi$, and its magnetic permeability $\alpha_{m}$ is $\alpha_{m}=2 b^{3} / 3 \pi$, see [16], Sec. 9.5. Assuming that the holes do not interact with each other, we conclude that a tangential magnetic field $H_{\theta}$ on the wall induces in holes the magnetic moment per unit area equal to $\alpha_{m} \rho H_{\theta}$ where $\rho=d^{-2}$ is the number of holes per unit area. For a magnetic layer of thickness $h$ the magnetic moment per unit area in the tangential field would be $H_{\theta}(\mu-1) / 4 \pi$, hence we find the correspondence between the product $(\mu-1) h$ and $\alpha_{m},(\mu-1) h \rightarrow 4 \pi \rho \alpha_{m}$. Similarly, a radial electric field $E_{r}$ induces in the holes the radial dipole moment per unit area equal to $\alpha_{e} \rho E_{r}$, whereas in the dielectric layer it would induces the dipole moment $E_{r}(\epsilon-1) / 4 \pi \epsilon$, hence $\left(1-\epsilon^{-1}\right) h \rightarrow 4 \pi \rho \alpha_{e}$. These two relations allow us to convert the surface impedance of the dielectric layer (13) into the surface impedance of holes

$$
\zeta_{\text {holes }}=-4 \pi \rho i k\left(\alpha_{e}+\alpha_{m}\right)=-\frac{4}{3} \rho i k b^{3}
$$

This result has been also recently found in Ref. [17]. 
Again, we see that this kind of surface impedance allows propagation of synchronous modes in the perforated pipe with the frequency

$$
\omega_{0}=c \sqrt{\frac{3}{2 a b^{3} \rho}} .
$$

This formula is applicable in the limit of noninteracting holes when the wavelength of the mode is much larger than the distance between the holes.

\section{Acknowledgements}

The author thanks K. Bane and V. Dolgashev for useful discussions.

This work was supported by the US Department of Energy, Office of Basic Energy Sciences, under contract \# DE-AC03-76SF00515.

\section{References}

[1] S. S. Kurennoy and G. V. Stupakov, Particle Accelerators 45, 95 (1994).

[2] K. L. F. Bane, C. K. Ng, and A. W. Chao, Report SLAC-PUB-7514, SLAC (1997).

[3] K. L. F. Bane and G. V. Stupakov, Tech. Rep. SLAC-PUB-8023, SLAC (December 1998), presented at International Computational Accelerator Physics Conference (ICAP 98), Monterey, CA, 14-18 Sep 1998.

[4] R. L. Gluckstern, Phys. Rev. A46, 1110 (1992).

[5] V. I. Balbekov, Tech. Rep. IFVE-93-55, IHEP, Protvino, Russia (April 1993).

[6] V. I. Balbekov, Tech. Rep. IFVE-93-56, IHEP, Protvino, Russia (April 1993).

[7] M. Dohlus, private communication, (1999).

[8] L. D. Landau and E. M. Lifshitz, Electrodynamics of Continuous Media, (Pergamon, London, 1960), 2nd ed. 
[9] A. V. Burov and A. V. Novokhatskii, Tech. Rep. BUDKERINP-90-28, Budker Inst. of Nucl. Physics, Novosibirk, Russia (February 1990).

[10] K.-Y. Ng, Phys. Rev. D42, 1819 (1990).

[11] A. Novokhatski and A. Mosnier, in Proceedings of the 1997 Particle Accelerator Conference (IEEE, Piscataway, NJ, 1997), pp. 1661-1663.

[12] K. L. F. Bane and A. Novokhatskii, Tech. Rep. SLAC-AP-117, SLAC (March 1999).

[13] G. V. Stupakov, Phys. Rev. ST Accel. Beams 1, 064401 (1998).

[14] M. Chatard-Moulin and A. Papiernik, IEEE Trans. Nucl. Sci. 26, 3523 (1979).

[15] S. Krinsky, in W. S. Newman, ed., Proc. International Conference on High-Energy Accelerators, Geneva, 1980, CERN, European Lab. for Particle Physics (Birkhäuser Verlag, Basel, Switzerland, 1980), p. 576.

[16] J. D. Jackson, Classical Electrodynamics (Wiley, New York, 1975), 2nd ed.

[17] S. Petracca, Tech. Rep. CERN-SL-99-003, CERN (February 1999). 